\title{
Subject-specific Models for the Analysis of Pathological FDG PET Data
}

\author{
Ninon Burgos ${ }^{1}$, M. Jorge Cardoso ${ }^{1,2}$, Alex F. Mendelson ${ }^{1}$, \\ Jonathan M. Schott ${ }^{2}$, David Atkinson ${ }^{3}$, Simon R. Arridge ${ }^{4}$, \\ Brian F. Hutton ${ }^{5,6}$, and Sébastien Ourselin ${ }^{1,2}$ \\ 1 Translational Imaging Group, CMIC, University College London, London, UK \\ 2 Dementia Research Centre, University College London, London, UK \\ 3 Centre for Medical Imaging, University College London, London, UK \\ 4 Centre for Medical Image Computing, University College London, London, UK \\ ${ }^{5}$ Institute of Nuclear Medicine, University College London, London, UK \\ ${ }^{6}$ Centre for Medical Radiation Physics, University of Wollongong, NSW, Australia
}

\begin{abstract}
Abnormalities in cerebral glucose metabolism detectable on fluorodeoxyglucose positron emission tomography (FDG PET) can be assessed on a regional or voxel-wise basis. In regional analysis, the average relative uptake over a region of interest is compared with the average relative uptake obtained for normal controls. Prior knowledge is required to determine the regions where abnormal uptake is expected, which can limit its usability. On the other hand, voxel-wise analysis consists of comparing the metabolic activity of the patient to the normal controls voxel-by-voxel, usually in a groupwise space. Voxel-based techniques are limited by the inter-subject morphological and metabolic variability in the normal population, which can limit their sensitivity.

In this paper, we combine the advantages of both regional and voxel-wise approaches through the use of subject-specific PET models for glucose metabolism. By accounting for inter-subject morphological differences, the proposed method aims to remove confounding variation and increase the sensitivity of group-wise approaches. The method was applied to a dataset of 22 individuals: 17 presenting four distinct neurodegenerative syndromes, and 5 controls. The proposed method more accurately distinguishes subgroups in this set, and improves the delineation of diseasespecific metabolic patterns.
\end{abstract}

\section{Introduction}

In current clinical practice, fluorodeoxyglucose positron emission tomography (FDG PET) is commonly used to diagnose dementias. FDG uptake reflects glucose consumption, which is reduced by synaptic dysfunction and neuronal degeneration [1]. Different neurodegenerative diseases affect different areas of the brain, and localising abnormalities is essential for differential diagnosis [2-4].

Cerebral glucose metabolism as measured by FDG PET can be quantitatively evaluated either regionally or on a voxel-by-voxel basis. In regional analysis, the regional standardised uptake value ratio, defined as the average uptake 
in a region of interest (ROI) relative to a reference region (commonly the pons or cerebellar grey matter), is compared with the regional SUVR expected in a normal control population. This analysis usually requires prior knowledge regarding the selection of relevant discriminant regions, limiting its use [5]. In voxel-wise analysis, a subject's PET image is usually aligned to a standardised group space to compare the metabolic activity of the spatially normalised scan on a voxel-by-voxel basis to a distribution obtained from normal control scans, e.g. to produce a Z-score [6]. Exploratory in nature, voxel-wise techniques require less prior information than regional SUVR analysis, but their sensitivity is limited by inter-subject variability in non-pathological tracer uptake, making pathological effects harder to detect [5]. Furthermore, there is theoretical uncertainty regarding how to appropriately resample PET data to the group space, how to match the PET point spread function with the group-wise model under linear and non-linear transformations, and how to map the PET data to the group space in a biologically plausible manner.

In this paper, we combine the advantages of both regional and voxel-wise approaches by analysing PET data in the original subject space (as in regional SUVR approaches) while still providing voxel-by-voxel statistics (as in groupwise approaches). This is achieved through a subject-specific PET model based on the propagation of morphologically-matched PET scans. The local morphological matching reduces both the bias and variance of the healthy patientspecific model, increasing sensitivity.

\section{Method}

In the following, 4 approaches analysing FDG PET images are explored: a regional analysis based on SUVR (section 2.3), a voxel-wise analysis in a group space (section 2.4), a voxel-wise analysis in the subject's space (section 2.5) and the proposed subject-specific analysis (section 2.6).

\subsection{Data}

Twenty-two sets of ${ }^{18}$ F-FDG PET and T1-weighted MRI brain images were used to evaluate the proposed methodology, here denoted validation dataset. Of these subjects, 5 were diagnosed with posterior cortical atrophy (PCA), 5 with semantic dementia (SD), 4 with progressive non-fluent aphasia (PNFA), 3 with logopenic progressive aphasia (LPA) and 5 were healthy controls. The T1-weighted magnetisation-prepared rapid gradient-echo $(3.0 \mathrm{~T}$; TE/TR/TI, $2.9 \mathrm{~ms} / 2200 \mathrm{~ms} / 900 \mathrm{~ms}$; voxel size $1.1 \times 1.1 \times 1.1 \mathrm{~mm}^{3}$ ) scans were acquired on a 3T Siemens Magnetom Trio scanner (Siemens Healthcare, Erlangen, Germany). PET images (voxel size $1.95 \times 1.95 \times 3.27 \mathrm{~mm}^{3}$ ) were acquired on a GE Discovery ST PET/CT scanner (GE Healthcare systems, Waukesha, WI) for

20 minutes, 30 minutes after injection of $185 \mathrm{MBq}{ }^{18} \mathrm{~F}$-FDG. The local ethics committee approved the study and all subjects gave written, informed consent. 
To create fully independent healthy population statistics and to demonstrate the applicability of the proposed patient-specific modelling strategy to multi-site data, a dataset of 29 healthy controls with T1-weighted MRI and ${ }^{18}$ F-FDG PET were selected from the ADNI2 database ${ }^{1}$. From here on, this dataset is referred to as the control dataset.

\subsection{Data preprocessing}

$\mathrm{T} 1$ images from the validation dataset were corrected for intensity non-uniformity following a nonparametric intensity non-uniformity normalisation method [7]. The T1 images from both the validation and control datasets were then parcellated into 143 different regions using a multi-atlas propagation and fusion algorithm implemented in NiftySeg [8]. PET images were intensity normalised using the mean uptake of the pons [9].

\subsection{Regional analysis}

Regional analysis consisted of computing the average standardised uptake in a region of interest to produce a regional SUVR (rSUVR) for each subject independently. This value is then compared with those obtained for normal controls (NC) by its expression as a Z-score, i.e.

$$
\mathrm{Z}_{\mathrm{rSUVR}}=\frac{\mathrm{rSUVR}-\mu_{\mathrm{rSUVR}}^{\mathrm{NC}}}{\sigma_{\mathrm{rSUVR}}^{\mathrm{NC}}},
$$

where $\mu_{\mathrm{rSUVR}}^{\mathrm{NC}}$ and $\sigma_{\mathrm{rSUVR}}^{\mathrm{NC}}$ are the mean and standard deviation of the 29 control subjects' rSUVRs.

\subsection{Voxel-by-voxel analysis in the group space}

For the voxel-by-voxel group-wise analysis, all image pairs from the control and the validation datasets were mapped to a common space defined through iterative group-wise non-rigid registration of the T1 data [10]. PET images were brought to the group space by composing the rigid transformations between paired PET and T1 images and the non-rigid transformation between the native T1 images and the group space. After resampling, PET images were smoothed with a Gaussian filter with $5 \mathrm{~mm}$ FWHM.

The 22 PET images of the validation dataset were compared with the 29 of the control dataset by expressing the value at each voxel $x$ as a Z-score

$$
\mathrm{Z}_{\text {group-wise }}(x)=\frac{I_{G}(x)-\mu_{G}^{\mathrm{NC}}(x)}{\sigma_{G}^{\mathrm{NC}}(x)},
$$

where $I_{G}$ corresponds to the subject's PET in the group space, and $\mu_{G}^{\mathrm{NC}}$ and $\sigma_{G}^{\mathrm{NC}}$ are the mean and standard deviation of the 29 subjects of the control dataset in the group space.

${ }^{1}$ Imaging data were provided by the Alzheimer's disease neuroimaging initiative (http://adni.loni.ucla.edu/). 


\subsection{Voxel-wise analysis in the subject's space}

Analysing the subject's PET image in its native space requires the control dataset to be transported to the target subject space. To do so, each of the 29 MRI images of the control dataset was deformed to the target image using an affine followed by a non-rigid registration [11]. The PET images of the control dataset, pre-aligned to the MRI images, were then mapped using the same transformation to the subject MRI image. The Z-score between the subject's PET and the entire control dataset aligned to the subject is defined as

$$
\mathrm{Z}_{\text {native }}(x)=\frac{I(x)-\mu^{\mathrm{NC}}(x)}{\sigma^{\mathrm{NC}}(x)},
$$

where $I$ corresponds to the subject's PET image, and $\mu^{\mathrm{NC}}$ and $\sigma^{\mathrm{NC}}$ are the arithmetic mean and standard deviation of the 29 ADNI2 controls mapped into the native subject space, respectively.

\subsection{Proposed voxel-wise subject-specific analysis}

This paper proposes a novel analysis framework that consists of creating a subject-specific healthy PET template. As in the previous approach (section 2.5), the control dataset is transported into the target subject space. A local image similarity measure, here the local normalised cross-correlation (LNCC), is then used to estimate the morphological similarity between the subject's MRI and the set of registered MRIs from the control dataset. To generate the subject-specific PET Z-score parameters, namely the patient-specific healthy-population mean and standard deviation, the set of registered PETs is locally selected and fused using a voxel-wise weighting scheme. As in [12], for all of the $N$ control dataset MRIs propagated to the target subject's MRI, the LNCC at each voxel is ranked across all images. This ranking, denoted $r_{n}(x)$ (with $x$ indexing the voxel and $n$ indexing the propagated MRI) is converted to a weight by applying an exponential decay function $w_{n}(x)=e^{-\beta r_{n}(x)}$, with $\beta=0.5$. Here, $w_{n}(x)$ represents the weight or contribution of subject $n$ at voxel $x$ to the patient specific model. For each of the $N$ subjects in the control dataset, let the $n^{t h}$ mapped PET image be denoted by $J_{n}$. The subject-specific Z-score model parameters $\left(I_{\mu}, I_{\sigma}\right)$ are obtained by a spatially varying weighted average

$$
\begin{aligned}
I_{\mu}(x) & =\frac{\sum_{n=1}^{N} w_{n}(x) \cdot J_{n}(x)}{\sum_{n=1}^{N} w_{n}(x)} \\
I_{\sigma}(x) & =\sqrt{\frac{N_{w}}{N_{w}-1} \frac{\sum_{n=1}^{N} w_{n}(x) \cdot\left(J_{n}(x)-I_{\mu}(x)\right)^{2}}{\sum_{n=1}^{N} w_{n}(x)}}
\end{aligned}
$$

where $N_{w}$ is the number of non-zero weights. Finally, the subject-specific Z-score is defined as

$$
\mathrm{Z}_{\text {subject-specific }}(x)=\frac{I(x)-I_{\mu}(x)}{I_{\sigma}(x)} .
$$




\section{Validation \& Results}

The ${ }^{18} \mathrm{~F}$-FDG PET images of the 22 subjects from the validation dataset were examined using the regional, group-wise, native and proposed subject-specific analyses. Comparisons were restricted to 8 clinically relevant regions (Figure 1, top) for the sake of brevity. These regions were selected to represent the areas where abnormal uptake, compared with controls, is expected for the four pathologies represented in our dataset:

- a frontal region, comprising the inferior frontal gyrus, precentral gyrus and anterior insula, was defined as relevant for PNFA subjects $[2,3]$;

- the anterior temporal region, comprising the hippocampus, amygdala and temporal pole, was defined as relevant for SD [3];

- the tempoparietal region, comprising the inferior parietal lobule, posterior middle and superior temporal gyri, was defined as relevant for LPA [3];

- finally, the occipital region, comprising the inferior, middle and superior occipital gyri, was defined as relevant for PCA [4].

Each hemisphere was analysed separately to account for left/right asymmetry.

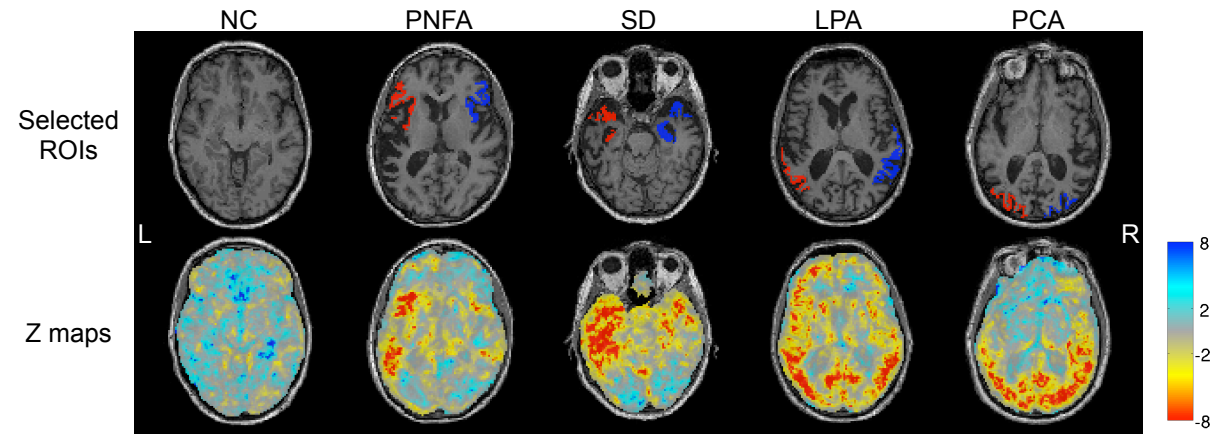

Fig. 1. T1 images overlaid with the selected ROIs (top) and the patient-specific Zscores (bottom) for a representative subject of each condition.

\subsection{Subject-specific Z-maps}

As a first evaluation of the proposed method, we visually assessed the subjectspecific Z-scores. Z-maps obtained for a representative subject of each condition are displayed in Figure 1 (bottom). A highly negative Z-score (red) indicates a reduced FDG uptake in the subject relative to the controls. Note that the Z-scores coincide with the regions where uptake abnormalities are expected for each pathology, and that the left/right asymmetries observed for the PNFA, SD, LPA and PCA subjects match the clinical syndromes: PNFA, SD and LPA subjects are expected to have greater left hypometabolism [3] while a greater right hypometabolism can be observed on subjects with PCA [4]. 

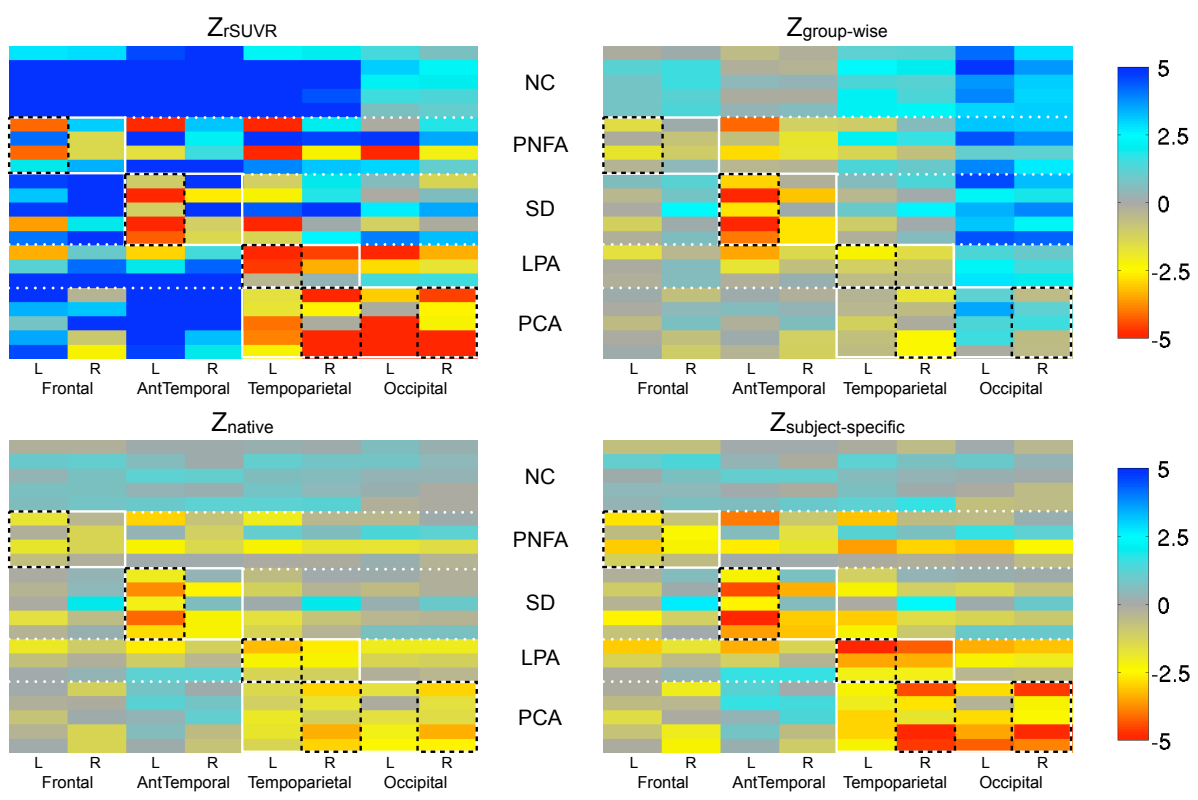

Fig. 2. Displayed above are the average Z-scores obtained for the rSUVR, group-wise, native and subject-specific methods in each ROI. The white boxes correspond to the regions where abnormal uptake is expected for each of the four pathologies while the black boxes indicate the predominant side when asymmetry is expected.

\subsection{Z-score comparison in predefined regions-of-interest}

The Z-scores obtained from the rSUVR, and average regional Z-scores obtained using the group-wise, native and proposed subject-specific methods in each ROI are displayed in Figure 2. We first note that, using the native and subject-specific methods, the controls have approximately zero Z-scores in all regions, while high Z-scores are observed in the occipital region with the group-wise method. We also note that, when using the proposed method, highly negative Z-scores are observed for each pathology in regions where a reduced uptake is expected when compared with healthy controls. Moreover, the left/right observed asymmetries match the clinical syndromes. These highly relevant Z-score results were stable for all the subjects in each pathological group. The same significance and stability was not observed in the native or group-wise methods.

A Wilcoxon-Mann-Whitney test was used to assess whether the different analysis methods were able to differentiate controls and subjects with pathology. Results showed that the $\mathrm{Z}_{\mathrm{rSUVR}}, \mathrm{Z}_{\text {native }}$ and the proposed $\mathrm{Z}_{\text {subject-specific }}$ scores could distinguish controls from each of the pathologies at a $5 \%$ significance level. With the $Z_{\text {group-wise }}$ score, only controls and PCA subjects were significantly different. These results suggest an improvement in sensitivity with the $Z_{\mathrm{rSUVR}}$, $Z_{\text {native }}$ and $Z_{\text {subject-specific }}$ scores when compared with the $Z_{\text {group-wise }}$ score. 


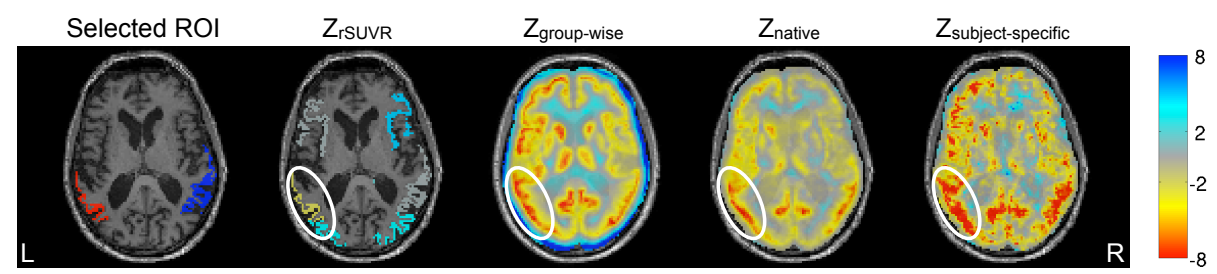

Fig. 3. Z-maps obtained with the different analysis methods for a subject with LPA. The white ellipses indicate the predominant side where abnormal uptake is expected.

Z-maps obtained for a representative LPA subject with the different analysis methods are displayed in Figure 3. We observe higher negative Z-scores in the relevant ROIs with the subject-specific approach than with the other methods.

\section{Discussion \& Conclusion}

In this paper, we analyse ${ }^{18}$ F-FDG PET data in the original subject space by providing voxel-wise statistics of normality/abnormality. The subject PET image is compared using a Z-score to a subject-specific PET model obtained through the propagation of morphologically-matched PET scans from a control dataset.

We first observed that, when analysing the PET images in the native subject space, the normal controls have low Z-scores in all regions, which was not always the case with the other methods (Figure 2). We note that the Z-scores obtained with the proposed method coincide with the regions where uptake abnormalities are expected for each of the pathologies studied and respect the left/right asymmetry (Figures $1 \& 2$ ). Finally, it appears that these highly negative Z-score results are stable across all subjects in each pathological group, which is not the case with the other methods (Figure 2).

The group-wise analysis seems strongly affected by uncertainties in the groupwise registration, driven by subjects with a large amount of atrophy (i.e. subjects with PCA), and the large variations in the normal population. Analysing the PET data in the native space avoids registering and resampling the subject PET into the group space. Furthermore, in the subject-specific method, only the most morphologically similar controls per target are selected to build the model, which reduces the variance of the normal population used for comparison in the Z-score, thus increasing the sensitivity. The low Z-scores systematically observed for the healthy subjects suggest that the subject-specific analysis is less prone to false positives. Finally, in contrast with rSUVR-based methods, the proposed analysis is not restricted to predefined regions.

While our method offers greater sensitivity, this is not its only purpose. It is our hope that, by disentangling the measurement of atrophy and metabolic decline, our method may provide a greater understanding of neurodegenerative diseases. Future work will involve validating the method on a larger dataset to provide more quantitative results. 
Acknowledgements This work was supported by an IMPACT studentship funded by Siemens and the UCL FES. Funding was received from the EPSRC (EP/H046410/1, EP/J020990/1, EP/K005278), the MRC (MR/J01107X/1), the EU-FP7 project VPH-DARE@IT (FP7-ICT-2011-9-601055), Alzheimer's Research UK, the Brain Research Trust, and the NIHR Biomedical Research Unit (Dementia). This work was also supported by funding from AVID Radiopharmaceuticals (a wholly owned subsidiary of Eli Lilly) and by researchers at the NIHR UCLH Biomedical Research Centre (including the High Impact Initiative).

\section{References}

1. Herholz, K.: PET studies in dementia. Annals of Nuclear Medicine 17(2) (2003)

2. Nestor, P.J., Graham, N.L., Fryer, T.D., Williams, G.B., Patterson, K., Hodges, J.R.: Progressive non-fluent aphasia is associated with hypometabolism centred on the left anterior insula. Brain 126(11) (2003) 2406-2418

3. Rabinovici, G.D., Jagust, W.J., Furst, A.J., Ogar, J.M., Racine, C.A., Mormino, E.C., O’Neil, J.P., Lal, R.A., Dronkers, N.F., Miller, B.L., Gorno-Tempini, M.L.: $\mathrm{A} \beta$ Amyloid and Glucose Metabolism in Three Variants of Primary Progressive Aphasia. Annals of Neurology 64(4) (2008) 388-401

4. Crutch, S.J., Lehmann, M., Schott, J.M., Rabinovici, G.D., Rossor, M.N., Fox, N.C.: Posterior cortical atrophy. The Lancet Neurology 11(2) (2012) $170-178$

5. Signorini, M., Paulesu, E., Friston, K., Perani, D., Colleluori, A., Lucignani, G., Grassi, F., Bettinardi, V., Frackowiak, R., Fazio, F.: Rapid Assessment of Regional Cerebral Metabolic Abnormalities in Single Subjects with Quantitative and Nonquantitative $\left[{ }^{18}\right.$ F]FDG PET: A Clinical Validation of Statistical Parametric Mapping. Neuroimage 9(1) (1999) 63-80

6. Drzezga, A., Grimmer, T., Riemenschneider, M., Lautenschlager, N., Siebner, H., Alexopoulus, P., Minoshima, S., Schwaiger, M., Kurz, A.: Prediction of Individual Clinical Outcome in MCI by Means of Genetic Assessment and ${ }^{18}$ F-FDG PET. Journal of Nuclear Medicine 46(10) (2005) 1625-1632

7. Sled, J.G., Zijdenbos, A.P., Evans, A.C.: A nonparametric method for automatic correction of intensity nonuniformity in MRI data. IEEE Transactions on Medical Imaging 17(1) (1998) 87-97

8. Cardoso, M., Wolz, R., Modat, M., Fox, N.C., Rueckert, D., Ourselin, S.: Geodesic Information Flows. In: MICCAI 2012. Volume 7511. (2012) 262-270

9. Minoshima, S., Frey, K.A., Foster, N.L., Kuhl, D.E.: Preserved Pontine Glucose Metabolism in Alzheimer Disease A Reference Region for Functional Brain Image (PET) Analysis. Journal of Computer Assisted Tomography 19(4) (1995) 541-547

10. Rohlfing, T., Brandt, R., Maurer, C.R., J., Menzel, R.: Bee brains, B-splines and computational democracy: generating an average shape atlas. Proc. IEEE Workshop Mathematical Methods in Biomedical Image Analysis (2001) 187-194

11. Modat, M., Ridgway, G.R., Taylor, Z.A., Lehmann, M., Barnes, J., Hawkes, D.J., Fox, N.C., Ourselin, S.: Fast free-form deformation using graphics processing units. Computer methods and programs in biomedicine 98(3) (2010) 278-84

12. Burgos, N., Cardoso, M.J., Thielemans, K., Modat, M., Pedemonte, S., Dickson, J., Barnes, A., Ahmed, R., Mahoney, C.J., Schott, J.M., Duncan, J.S., Atkinson, D., Arridge, S.R., Hutton, B.F., Ourselin, S.: Attenuation Correction Synthesis for Hybrid PET-MR Scanners: Application to Brain Studies. IEEE Transactions on Medical Imaging 33(12) (2014) 2332-2341 